of the IBP criteria for diagnosis of IBP according to the local rheumatologist, diagnosis of axial SpA by local rheumatologist, diagnosis of axial SpA by central assessment, and concordant diagnosis of axial SpA by both local and central assessment.

Results: We recruited 185 patients ( $48.6 \%$ male, mean age 27.6 years, mean back pain duration 7.0 years) with iritis $(30.3 \%)$, psoriasis (17.3\%), Crohn's $(38.4 \%)$ and ulcerative colitis $(18.9 \%)$. IBP and/or axial SpA were considered present in $65.2 \%$ and $47.3 \%$, respectively, of all patients by the local rheumatologist. By central assessment axial SpA was considered present in $32.7 \%$ while amongst patients with a concordant diagnosis by both local and central assessment $37.2 \%$ were considered to have axial SpA. Sensitivity was comparatively high for all IBP criteria but specificity was poor. The Berlin criteria consistently performed best while the Calin criteria consistently performed worst (Table).

\begin{tabular}{lccccc}
\hline Gold standard & IBP criteria & $\begin{array}{c}\text { Sensitivity } \\
(\%)\end{array}$ & $\begin{array}{c}\text { Specificity } \\
(\%)\end{array}$ & LR+ & LR- \\
\hline IBP by local rheumatologist & ASAS & 83.8 & 58.8 & 2.03 & 0.28 \\
& Berlin & 88.9 & 76.5 & 3.78 & 0.15 \\
& Calin & 94.0 & 37.3 & 1.50 & 0.16 \\
Axial SpA by local rheumatologist & ASAS & 82.9 & 44.9 & 1.50 & 0.38 \\
& Berlin & 84.3 & 51.3 & 1.73 & 0.31 \\
Axial SpA by central assessment & Calin & 90.0 & 19.2 & 1.11 & 0.52 \\
& ASAS & 80.0 & 37.5 & 1.28 & 0.53 \\
Axial SpA by central and local assessment & Berlin & 80.0 & 38.9 & 1.31 & 0.51 \\
& Calin & 94.3 & 20.8 & 1.19 & 0.27 \\
& ASAS & 86.2 & 44.9 & 1.56 & 0.31 \\
& Berlin & 86.2 & 51.0 & 1.76 & 0.27 \\
& Calin & 93.1 & 22.4 & 1.20 & 0.31 \\
\hline
\end{tabular}

Conclusions: IBP criteria lack specificity for rheumatologist diagnosed IBP or axial $\mathrm{SpA}$ but the Berlin criteria have consistently better performance.

Disclosure of Interest: None declared

DOI: 10.1136/annrheumdis-2017-eular.5151

\section{SAT0429 THE PERFORMANCE OF MAGNETIC RESONANCE IMAGING USING THE VIBE TECHNIQUE TO DETECT STRUCTURAL CHANGES IN PATIENTS WITH EARLY AXIAL SPONDYLOARTHRITIS IN COMPARISON TO CONVENTIONAL RADIOGRAPHY AND COMPUTED TOMOGRAPHY}

$\underline{\text { X. Baraliakos }}{ }^{1}$, F. Hoffmann ${ }^{1}$, X. Deng ${ }^{2}$, Y. Wang ${ }^{2}$, F. Huang ${ }^{2}$, J. Braun ${ }^{1}$ ${ }^{1}$ Rheumazentrum Ruhrgebiet, Herne, Germany; ${ }^{2}$ Department of Rheumatology, Chinese PLA General Hospital, Beijing, China

Background: Magnetic resonance imaging (MRI) is the gold standard for detection of inflammation in the sacroiliac joints (SIJ) of patients (pts) with axial spondyloarthritis (axSpA), while for chronic, structural changes (erosions, sclerosis and ankylosis) conventional radiographs (CR) and computed tomography (CT) are often preferred. The 3D volumetric interpolated breath-hold sequence (VIBE) is an MRI technique, easy to acquire in daily practice, that can visualize cartilage especially well because of its good contrast to synovial tissue.

Objectives: To compare the ability of the VIBE technique to detect structural changes in comparison to $\mathrm{CR}$ and $\mathrm{CT}$ in SIJs of axSpA patients in relation to symptom duration and age.

Methods: Complete sets of MRI (T1 and VIBE techniques), CT and CR of SIJs of 109 AS patients were available. Two readers evaluated all images independently, blinded to demographic data and in separate sessions for each technique. The assessment of lesions was performed based on SIJ-quadrants (SQ) to score erosions, sclerosis and ankylosis (SIJ-halves). Lesions were counted as positive if both readers were in agreement. Comparisons between MRI techniques were performed by Wilcoxon-test. Linear regression analysis was used to evaluate the influence of age and disease duration on the occurrence of different structural lesions by modeling the differences in the number of lesions in different imaging techniques as dependent variable.

Results: The mean age \pm standard deviation was $45.3 \pm 13.9$ years $(\mathrm{y}), 55$ pts $(50.5 \%)$ were aged $\leq 45 \mathrm{y}, 67.9 \%$ male, $82.3 \%$ HLA-B27+, 58 pts $(53.2 \%)$ had a disease duration $\leq 3 y$. Agreement for positive and negative findings between MRI and CT was generally high ( $>80 \%$ of SQs in all subgroups) and agreement between readers for all techniques and lesion types was excellent (ICC=0.979-0.997).

Overall, MRI detected significantly more $S Q$ with erosions in pts $\leq 45 y \quad(n=134)$ and in pts with disease duration $\leq 3 y(n=125)$ as compared to CT $(n=91, p=0.002$ and $n=90, p=0.003$, respectively) and in pts with age $\leq 45 y \quad(n=61, p<0.001)$ as compared to $C R$, while there were no differences between MRI and CT in pts. $>45 y$ or disease duration $>3 y$. Linear regression analysis showed that MRI was superior in the detection of erosions in younger ages as compared to CT $(\mathrm{B}=0.032, \mathrm{p}=0.001)$.

However, CT detected significantly more SIJ halves with ankylosis in all subgroups and more SQ with sclerosis in pts with disease duration $\leq 3 y$ ( $n=64$ vs. $n=37$, respectively, $p=0.006$ ), and it also detected more $S Q$ with sclerosis in pts $>45 y$ $(n=67$ vs. $n=38, p=0.001)$ and disease duration $>3 y(n=64$ vs. $n=40, p=0.003)$ as compared to MRI, while no differences were found in the assessment of ankylosis. Conclusions: $\mathrm{MRI}$ in the $\mathrm{T} 1$ and VIBE technique is more sensitive in the detection of erosions as compared to CT and CR in axSpA pts with short disease duration and younger age. This is due to its ability to identify structural damage in the SIJ cartilage that has not yet extended to the underlying bone. These differences are not found in pts with longer disease duration or older age. This data suggests a more prominent role for MRI also for the early detection of structural changes in the SIJ of axSpA pts.

Disclosure of Interest: None declared

DOI: 10.1136/annrheumdis-2017-eular.6501

\section{SAT0430 THE RELATIONSHIP BETWEEN EXOSOMAL MIRNA21-5P AND ANKYLOSING SPONDYLITIS}

Y. Huang, T. Li, Z. Huang, W. Deng, S. Zheng, Z. Huang. Guangdong No. 2 Provincial People's Hospital, Guangzhou, China

Background: Ankylosing Spondylitis (AS) affects human health seriously, which is difficult to diagnose in the early stages. It is reported that MicroRNAs (miRNAs) may serve as novel biomarkers for AS. Exosome can function as vehicles to deliver miRNAs in body fluids including saliva and plasma. Our previous study shows that exosomal miRNA21-5P is higher expressed in AS patients, compared with healthy subjects. However, the relationship between exosomal miRNA21-5P and AS has yet to be determined.

Objectives: The aim of the present study is to explore the relationship between exosomal miRNA21-5P and AS.

Methods: AS patients who fulfilled the modified New York criteria were enrolled for this study. Healthy subjects were also enrolled as control group. BASDAI, $\mathrm{BASFI}, \mathrm{C}$-reactive protein (CRP) and erythrocyte sedimentation rate (ESR) were evaluated. Quantitative reverse-transcription PCR (qRT-PCR) was used to confirm the expression of exosomal miRNA21-5P, and receiver-operator characteristic (ROC) curve was used to evaluate the diagnostic value of exosomal miRNA21-5P for AS. According to the cut off value, AS patients were divided into exosomal miRNA21-5P low value group ( $<$ cut off value) and exosomal miRNA21-5P high value group ( $\geq$ cut off value), and the difference of AS patient's clinical characteristics between the two group were explored.

Results: Twenty healthy subjects and 38 AS patients were enrolled in the study. The qRT-PCR results indicated that the expression level of exsomal miRNA21-5P in AS patients was $(2.041 \pm 0.975)$ times higher than that of healthy subjects. ROC curve analysis showed that exsomal miRNA21-5P had significant diagnostic value for AS with the AUC of 0.809 (Cl95\%: 0.691-0.921). In addition, the cut off value was 1.310 , with the specifity of $80.0 \%$ and sensitivity of $76.32 \%$. According to the cut off value of exsomal miRNA21-5P, AS patients were divided into the low exsomal miRNA21-5P group $(<1.310)$ and the high exsomal miRNA21-5P group ( $\geq 1.310$ ). Among the 38 AS patients, 12 cases were in the low exsomal miRNA21-5P group and 26 cases were in the high exsomal miRNA21-5P group. Comparations of the clinical characteristics of the two groups showed that BASDAI, BASFI, CRP and ESR were significantly increased in exsomal miRNA21-5P high value group.

Conclusions: Exsomal miRNA21-5P was significantly increased in AS patients, which may be used as a biomarker for AS.

References:

[1] Atayde VD, Aslan H, Townsend S, Hassani K, Kamhawi S, Olivier M. Exosome.

[2] Secretion by the Parasitic Protozoan Leishmania within the Sand Fly Midgut. Cell Reports, 2015, 13 (5): 957-67.

Disclosure of Interest: None declared

DOI: 10.1136/annrheumdis-2017-eular.6836

\section{SAT0431 THE RELATIONSHIP OF SEASONAL VARIATION, THE ONSET AND THE SYMPTOMS OF ANKYLOSING SPONDYLITIS MEASURED BY SELF-ADMINISTRATED QUESTIONNAIRES IN CHINESE PATIENTS}

Y. Jiang, M. Yang, Z. Lin, Q. Wei, S. Cao, J. Gu. Rheumatology, the Third Affiliated Hospital of Sun Yat-sen University, Guangzhou, China

Background: Ankylosing spondylitis (AS) is a chronic inflammatory disease which mainly involves sacroiliac joints, resulting in pain, functionally limitation and even less life expectancy. Seasonal variation was found in rheumatoid arthritis and gout $[1,2]$. No reports had been focused on seasonal variation of AS onset or symptoms in AS patients.

Objectives: Our study was to investigate the relationship of seasonal variation and the onset and symptoms of AS in Chinese patients.

Methods: Adult AS patients diagnosed with the modified New York criteria for AS whose disease duration was over 2 years were enrolled from several provinces all over China. Participants were required to complete a set of questionnaires and examinations, including demographic and clinical information. Questions included "in which season(s) did you have the initial symptoms of AS", and "in which season(s) were the symptoms aggravated/improved". The Statistical Package for Social Sciences (SPSS) software version 21 was used for all data management and analysis.

Results: Of all the 859 AS patients, $75.1 \%$ were male patients. $47.8 \%$ were married. Mean age was $30.60 \pm 9.50$ years. Mean disease duration was $7.43 \pm 6.92$ years. $27.7 \%$ of the patients had an onset of the disease in summer, while the lowest incidence happened in autumn $(12.5 \%, p<0.05) .29 .6 \%$ of the patients could not recall the exact season. $29.5 \%$ of the patients' symptoms got worse in winter, 